\title{
Development of the Smart Mat with loT Applied Technology
}

\section{Jung-Sook $\mathrm{Kim}^{1}$ and Junho Jeong ${ }^{2}$}

${ }^{1}$ School of CIT Convergence, Kimpo University, Korea

${ }^{2}$ Department of Computer Science and Engineering, Kongju National University, Korea

\section{ljfis}

Received: Nov. 20, 2019

Revised : Nov. 22, 2019

Accepted: Dec. 4, 2019

Correspondence to: Jung-Sook Kim (kimjs@kimpo.ac.kr)

(C)The Korean Institute of Intelligent Systems

(c) This is an Open Access article distributed under the terms of the Creative Commons Attribution Non-Commercial License (http://creativecommons.org/licenses/ by-nc/3.0// which permits unrestricted noncommercial use, distribution, and reproduction in any medium, provided the original work is properly cited.

\begin{abstract}
In this study, we developed a smart mat with Internet of Things applied technology. The smart mat system composed of the hardware and software. The hardware contains multiple sensors connected to wireless network to transmit data from sensors to remote server. Also, the system can set the temperature at the optimal sleeping condition using a microcomputer. Furthermore, it can control the precision temperature controller at the unit of 1 grade and can control the controller at the minimum grade of 25-55. And the sensing data from smart mat are for remote monitoring of the elderly living alone or healthcare users, and a system model that can collect data on bedtime, wakeup time, and sleep quality. We developed an algorithm which could decides the status of sleep according to data from the sensors of the smart mat. There are nine types of status flags, which have the values from 1 to 9 . This system can process the recorded contents and send the data to the smartphone application of the caregivers in real time for remote monitoring. Also, we developed the management system on the web to check the device status, permission, custom information, user information, and raw data.
\end{abstract}

Keywords: Smart Mat System, Washable and Eco-friendly Materials, Remote Monitoring System, Sleeping Hours, Internet of Things, Healthcare, Biosensor

\section{Introduction}

In today's aging society, the need for effective health care for the senior citizens living alone is increasing greatly. Therefore, a healthcare system must be established to cope with daily health checks and emergency situations. Of various requirements, sleep is the most important part of health because we spend one quarter of a day's time sleeping. Especially, Korean many senior citizens use a mat to sleep and seat at home. The mat could provide the customized temperature for them. If the user wants to warm mat to sleep then the senior citizen can control the mat temperature. And many kinds of mat are manufactured in Korea.

The manufacturing industry uses Internet of Things (IoT) services, in which IoT is expected to have improved productivity. The mat factories try to make a smart mat which is applied IoT technology. A smart mat system uses at home. And the smart mat system can integrate into a smart home. Currently, smart home technologies are controlled by smartphones. However, senior citizens who need health care are having difficulty adapting to smartphone systems. Therefore, if their children or caregivers can monitor users' daily management of sleeping and cleaning time through smartphone remotely, they will be able to respond to emergencies within the golden hour of health. While the federal and local governments are concerned about 
the rapidly growing capacity of senior citizens to manage health and cope with crises, it is difficult for them to efficiently cope with sudden emergencies because of a lack of budget and manpower. Therefore, it needs to establish a remote healthcare system to cope with routine health checks and emergencies. Additionally, the paradigm is shifting from medical services centered in hospitals, to prevention and consumer-oriented care, where individuals manage their own health when diseases occur [1-6].

This study proposes an IoT applied technology smart mat system model which can access a remote system to monitor the user's sleeping status. The developed smart mat system is composed of the hardware and software. The hardware is made by eco-friendly and washable material. The developed software are two kinds applications. The one is the mobile application which is for remote monitoring. The another application operates on the web and it is for controlling the smart mat system. The structure of this study is as follows: Section 2 introduces related work, Section 3 presents the proposed system model and introduces Android-based mobile applications, and Section 4 describes the experimental results. Finally, Section 5 discusses conclusions and examine the direction of future studies.

\section{Related Work}

Essentially, smart home means human-centered smart life environment where information communication technology is fused into residence and national benefits, welfare enhancement and safe life are available. With such smart home infra, home appliances and sensors are connected by heterogeneous wire or wireless network and protocol, and operating system and software are mixed for this.

According to the Korea Association of Smart Home, the size of local smart home market exceeded 10 trillion won in 2015, with an annual average of growth by $20 \%$ or more, expecting around 19 trillion won of market in 2018. For example, some people use their smartphones to lose weight by connecting them to their scales, and some use smart watches to get messages or listen to music while keeping their smartphones in their bags. These are called 'IoT products' and are now evolving to provide new services by connecting various objects instead of using just one product. IoT services are being introduced in the manufacturing industry, in which IoT is expected to have improved productivity.

\section{IoT-Based Smart Mat System}

\subsection{System structure}

The developed system composed of the hardware and software. The hardware system is a smart mat, device management and database server. The software is two kinds. The one is a mobile monitoring application and another is for device management application. There are also the users of the mat, namely senior citizens, and users of the monitoring application, namely caregivers. The smart mat includes sensing data control that measures sleeping time and quality using gyro sensors, control devices that control the temperature of the mat, and access the Internet using Wi-Fi. The sensing data is sent to the event analysis application server via HTTP-based requests. Furthermore, we developed the smart mat to communicate the signal processing of human body sensors in the standardized Wi-Fi module. The sensor signal is received using the $\mathrm{Wi}-\mathrm{Fi}$ module with MCU(ESP-8266), and we manufactured PCB at minimum to equip it in the mat by designing it to communicate with Wi-Fi. We developed a system that is easy to install, even for the elderly, by developing the data communication to use the ESP-8266 Wi-Fi module and communication system with Wi-Fi router with fixed SSID/PW.

\subsection{Algorithm}

The sleeping stage is determined by the number of tossings and is measured every $10 \mathrm{~min}$ and saved. Bringing the user's fixed time data to be analyzed from the database, the continued sleep cycle is cut and analyzed. If several continued sleep cycles appear in the fixed time, the algorithm is repeated and each sleep cycle analyzed. After analyzing the data brought in and cut in continued sleep, the initial hour of continued sleep is saved as startTime, and the state of sleep stage is saved as 0 . Each data of continued sleep is analyzed in order, and the following process is repeated until the final data of continued sleep appears. If the data is not within $10 \mathrm{~min}$ from startTime, the actual state is saved, and the state to process for $10 \mathrm{~min}$ is generated. The standard is to add $10 \mathrm{~min}$ to the startTime. If tossing is sensed in $1 \mathrm{~min} 30 \mathrm{~s}, 1 \mathrm{~min}$ is added to the sleeping stage. The continued sleep cycles are analyzed by repeating the above process, and it is possible to analyze the sleep stage at the interval of $10 \mathrm{~min}$. The measuring algorithm is as follows:

$$
\begin{aligned}
& \text { Algorithm_MeasureSleepingHours() \{ } \\
& \text { While (1) }\{
\end{aligned}
$$


Bring raw data and ownership data from database;

Extract the continued sleep information

startTime = Initial time of continued sleep;

Save the initial point of continued sleep information as 0 state of sleep stage;

If (Is this the final data of continued sleep) \{

If (Has been the data sensed again in 1 minute and 30 seconds)

toppingCount $=$ toppingCount +1 ;

else

Save the state of 10 minutes;

\}

else if (Is it the data in 10 minutes from startTime) \{

if (Has been the data sensed again in 1 minute and

30 seconds)

toppingCount $=$ toppingCount +1 ;

else \{

Save the state of 10 minutes;

Generate a new state to save the next 10 minutes sleep state

stateTime $=$ startTime $+10 ;$

\}

Save the final point of continued sleep information as the state of sleep stage 9 ; \}

\subsection{Smart Mat}

The developed smart mat material is to be washable and ecofriendly. We also developed the human body sensing pressure sensor. The washable smart mat with a sensor can measure the sleeping hours and quality of sleep. The developed materials are fixed and unified, so as not to be contracted, relaxed, or damaged when washing, by including several materials in thermosetting mode. The human body sensing pressure sensor contacts the human body in the sleeping mat. The conductivity was processed in the felt to maintain the soft mat touch and cushion sense, and the human body sensing pressure sensor was designed and developed by perforating PU foam. A system can set the temperature at the optimal sleeping condition using a microcomputer. Furthermore, it can control the precision temperature controller at the unit of 1 grade and can control the controller at the minimum grade of 25-55. In addition, it provides the safety and energy saving function with automatic power interruption for $15 \mathrm{~h}$ of sleeping at maximum and supplies an alarm system of automatic level control.

The system contains a three-point touch type pressure sensor using the conductive fiber, and the human body sensor can distinguish the state of sleeping or sitting. The touch sensor using pressure can distinguish the state of sitting on bed during the sleeping hour and daily life or a pet's touch because it was designed to sense the human body when touching more than two times the shoulder, hip, and leg. It senses the electric signal in the superior and inferior plate using pressure by installing the conductive fiber in the superior and inferior plate and perforating the insulated pad. The sensor was designed and manufactured for the electric signal to be well-versed only by pressing more than two pads by composing the superior plate of the other three divided pads. Figure 2 depicts the 3 -point sensor.

\subsection{Remote Monitoring}

The remote monitoring system can communicate with the event analysis server using wireless network to transmit the collected data to the event analysis server, which processes the data to the monitoring application. At first, we analyze the status of sleep according to data which is received from the sensors of the smart mat. There are nine types of status flags, which have the values from 1 to 9. Through this study, we developed a REST API to send and receive data through HTTP-based requests. The following table shows the APIs.

\section{Experimental Results}

Figure 1 shows a hardware system for connection between the sensors and wireless communication to send the data.

The system contains a three-point touch type pressure sensor that uses the conductive fiber, and the human body sensor can distinguish the state of sleeping or sitting. Figure 2 shows a three-point touch pressure sensor.

The developed mobile application operates on the Android environment. The application provides many functions for remote monitoring and system management. For remote monitoring from caregivers in real-time, it provides a daily sleeping time, weekly sleeping time and monthly sleeping time. And to manage the system effectively in the web, it provides a user search, the status of users, device attachment, ownership, permission and accessing raw data. The user's sleeping hour is 
Table 1. APIs

\begin{tabular}{|c|c|c|c|c|}
\hline $\begin{array}{l}\text { API } \\
\text { description }\end{array}$ & Method & $\begin{array}{l}\text { Request } \\
\text { URL }\end{array}$ & Response & $\begin{array}{l}\text { Response } \\
\text { description }\end{array}$ \\
\hline \multirow{2}{*}{$\begin{array}{l}\text { Search of } \\
\text { all users }\end{array}$} & \multirow[t]{2}{*}{ GET } & \multirow[t]{2}{*}{ /user } & 200 & - \\
\hline & & & 400 & - \\
\hline \multirow[t]{3}{*}{ User creation } & \multirow[t]{3}{*}{ POST } & \multirow[t]{3}{*}{ /user } & 201 & Creation \\
\hline & & & 400 & - \\
\hline & & & 409 & $\begin{array}{l}\text { Already } \\
\text { existence } \\
\text { user ID }\end{array}$ \\
\hline \multirow{3}{*}{$\begin{array}{l}\text { Information } \\
\text { retrieval of } \\
\text { users }\end{array}$} & \multirow[t]{3}{*}{ GET } & \multirow[t]{3}{*}{ /user/ $\{$ user_id $\}$} & \} 200 & - \\
\hline & & & 400 & - \\
\hline & & & 404 & $\begin{array}{c}\text { Not found } \\
\text { user }\end{array}$ \\
\hline \multirow{3}{*}{$\begin{array}{l}\text { Information } \\
\text { update of } \\
\text { users }\end{array}$} & \multirow[t]{3}{*}{ PUT } & \multirow[t]{3}{*}{ /user/\{user_ic } & 200 & - \\
\hline & & & 400 & - \\
\hline & & & 404 & - \\
\hline \multirow[t]{2}{*}{ User deletion } & \multirow{2}{*}{\multicolumn{2}{|c|}{ DELETE /user/ $\{$ user_id $\}$}} & \} 204 & Deletion \\
\hline & & & 400 & - \\
\hline \multirow[t]{4}{*}{ Login } & \multirow[t]{4}{*}{ POST } & \multirow[t]{4}{*}{$/ \operatorname{login}$} & 201 & - \\
\hline & & & 400 & - \\
\hline & & & 401 & $\begin{array}{c}\text { Wrong } \\
\text { password }\end{array}$ \\
\hline & & & 404 & $\begin{array}{c}\text { Not found } \\
\text { user }\end{array}$ \\
\hline
\end{tabular}

shown as a graph. The daily status shows different shades of the colors, and the number of tosses and turns over the whole sleep cycle depending on each sleeping status. The date can be selected pressing the calendar button in the middle. Using a circular graph depending on the degree of tosses and turns, information can be conveyed to users by different shades of the

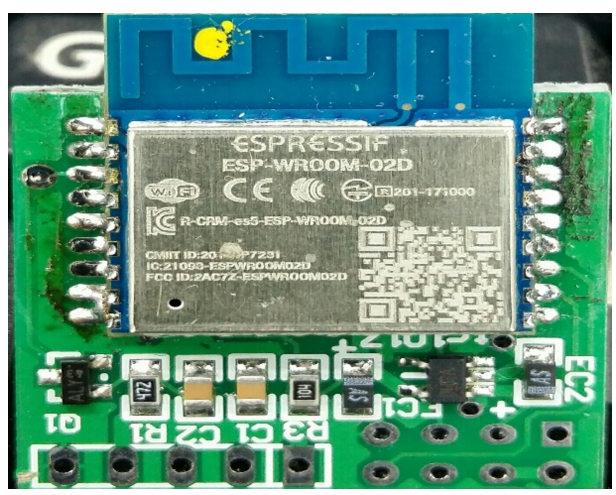

Figure 1. System to connect.

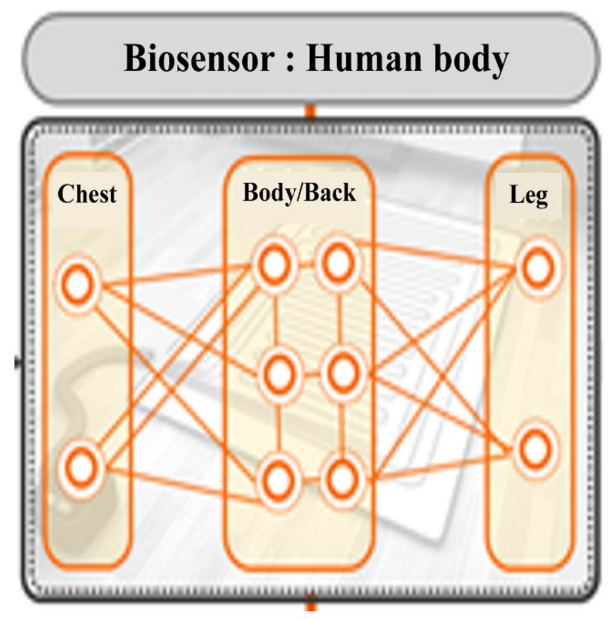

Figure 2. 3-point biosensors.

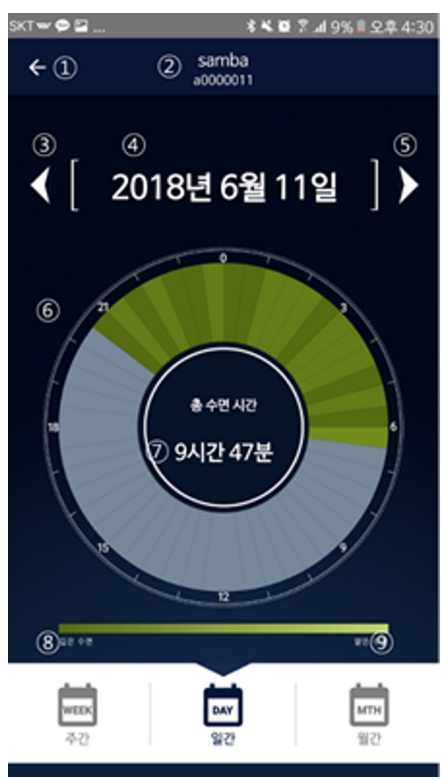

Figure 3. Daily data result.

colors. Counting the number of tosses and turns in each period and dividing sensed time by $10 \mathrm{~min}$, the sleeping state can be determined. When the number of tosses and turns is over a specific degree, it can be regarded as shallow sleeping. For the efficiency of the algorithm, process the next $10 \mathrm{~min}$ when the degree is over specific times already and regarded as shallow sleeping. Figure 3 shows the daily result because the middle button on the bottom is selected. The $\mathrm{x}$-axis represents a date and $y$-axis means sleeping hours

Weekly and monthly results are expressed by a bar graph with a line graph. The user can choose the weekly data screen pressing the first menu bar button at the bottom of the screen, as shown in Figure 4. 


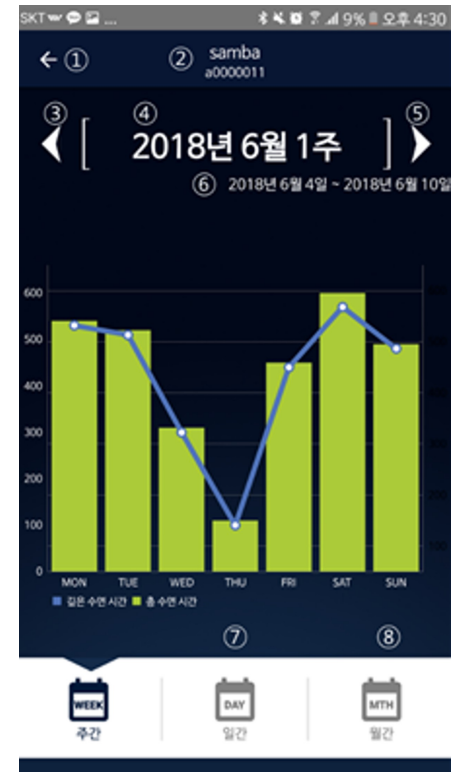

Figure 4. Weekly data result.

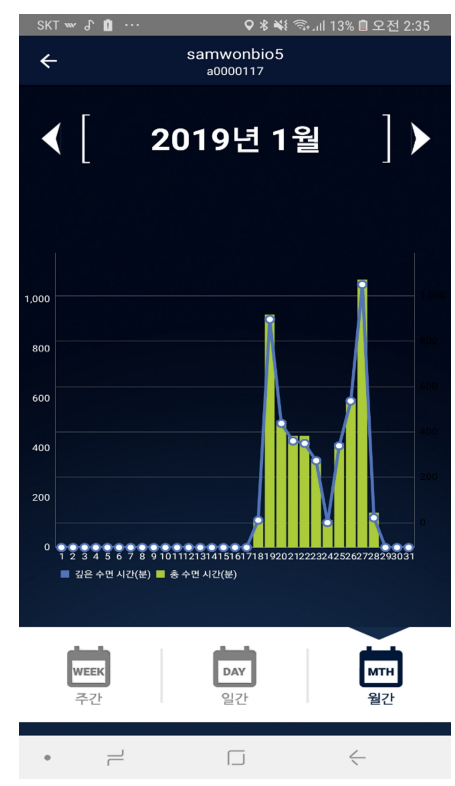

Figure 5. Monthly data result.

Figure 4 shows the weekly data result.

Figure 5 shows the monthly data result.

Figure 6 shows the login screen to remotely monitor sleep hours. We provide two types of login methods for senior citizens users. Logging in to the monitoring application first, the smart mat system can store the login information. After the initial login to the monitoring application, the login information should load automatically. This automatic login function is convenient for elderly users who may have difficulty memorizing

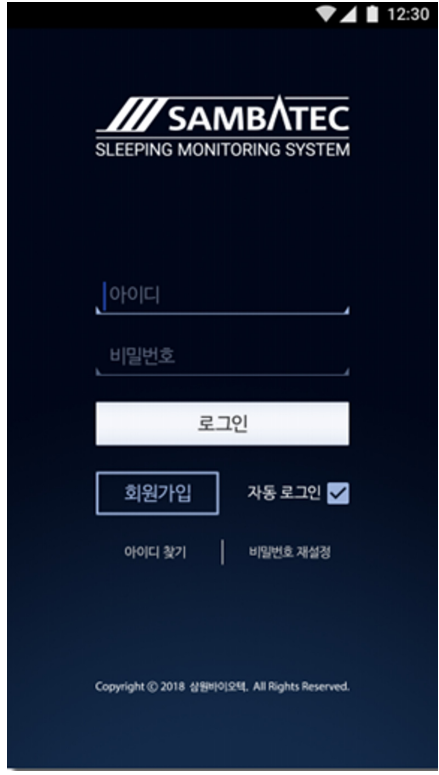

Figure 6. Login.

an ID and password. A simple input method was required; thus, we developed the automatic login function.

In addition, we designed and developed the new device registration screen that registers new user device information and information about user's device to register the relationship between them. Therefore, there is no need to register the device separately. It uses ID overlap confirmation and can remind the user about the entered value using a placeholder. Figure 7 shows the new device registration screen and figure 8 shows the information of the registered device information in the database.

Figure 8 shows the information about devices which is registered into the smart mat system.

The performance of the smart mat system to guarantee are verified that the smart mat system is convenient and correct. The testing results are that the internal sensor of smart mat is operating well and that the sensing data are transmitted into the server. Figure 9 depicts the smart mat.

The following tables show the testing result in the Korea Testing Certification (KTC). The test was repeated five times, and the five results follows:

\section{Conclusion}

In this study, we developed a smart mat with Internet of Things applied technology. The smart mat system composed of the hardware and software. The hardware contains multiple sensors connected to wireless network to transmit data from sensors to 


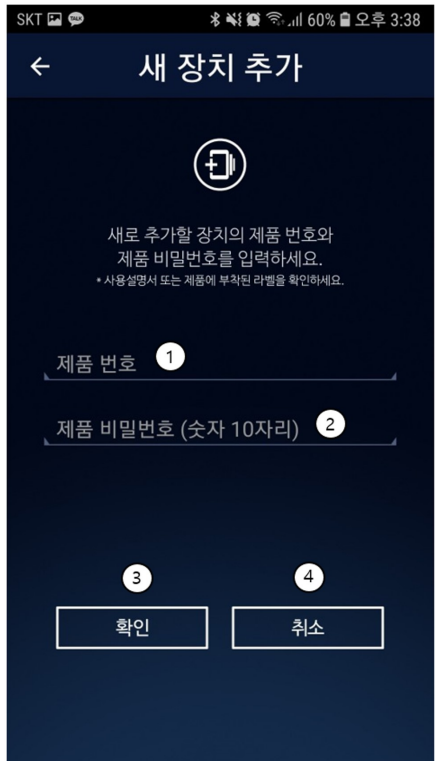

Figure 7. New device registration.

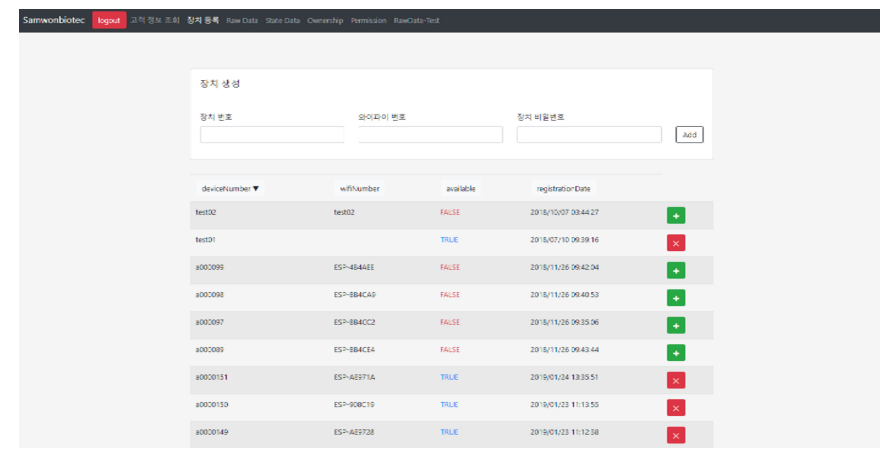

Figure 8. Device information.

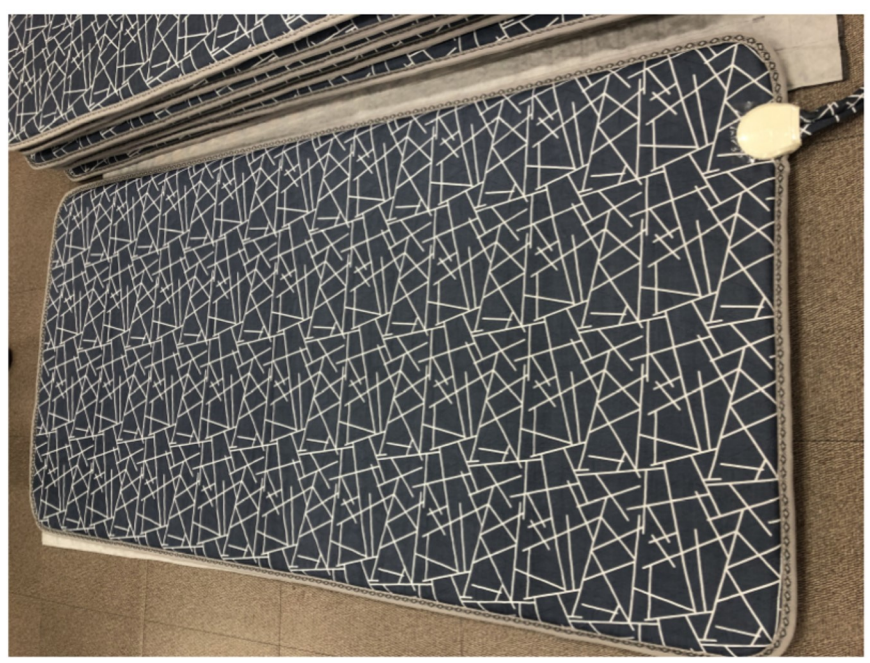

Figure 9. The smart mat.

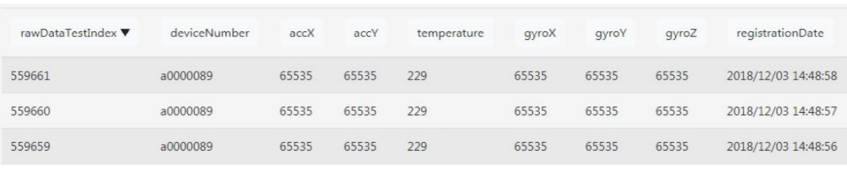

(a)

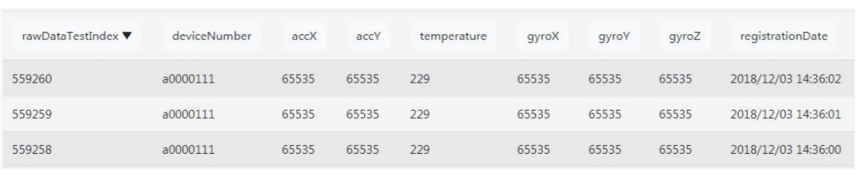

(b)

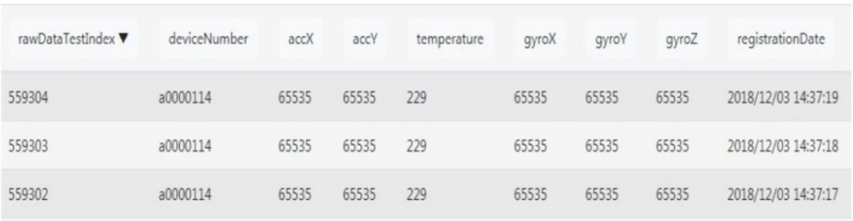

(c)

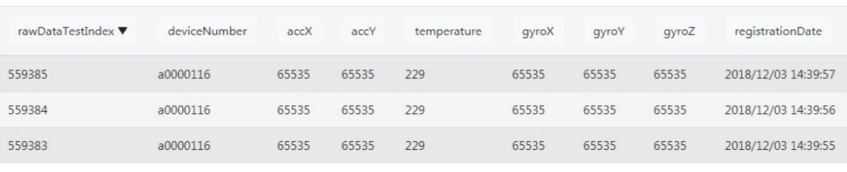

(d)

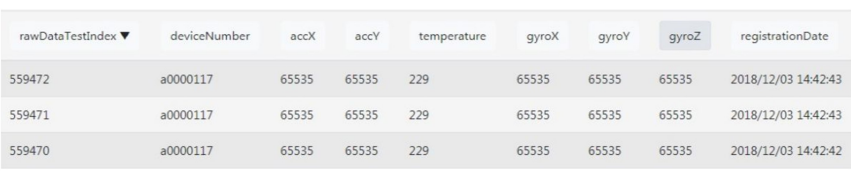

(e)

Figure 10. (a) The first time testing result, (b) the second time testing result, (c) the third time testing result, (d) the fourth time testing result, and (e) the fifth time testing result.

remote server. It uses an eco-friendly and washable material. Also, the system can set the temperature at the optimal sleeping condition using a microcomputer. Furthermore, it can control the precision temperature controller at the unit of 1 grade and can control the controller at the minimum grade of 25-55. And the sensing data from smart mat are for remote monitoring of the elderly living alone or healthcare users, and a system model that can collect data on bedtime, wakeup time, and sleep quality. And, we developed an algorithm which could decides the status of sleep according to data which is received from the sensors of the smart mat. There are nine types of status flags, which have the values from 1 to 9 . This system can process the recorded contents and send the data to the smartphone application of the caregivers in real time for remote monitoring. Also, we developed the management system on the web to check the device status, permission, custom information, user 
information, and raw data. Based on the information to be collected, we developed a simple application for checking sleep information. The data collected from the smart mat is displayed in the application with different kinds graph for easy readability. The remote monitoring system can remind the user about the entered value using a placeholder or can automatically log the user in. In the future, we will apply this system in the field for the senior citizens living alone. Furthermore, we will upgrade the smart mat system using machine learning for more detailed result analysis from raw data.

\section{Conflict of Interest}

No potential conflict of interest relevant to this article was reported.

\section{Acknowledgements}

The work (Grants No. C0564096) was supported by the Business for Cooperative R\&D between Industry, Academy, and Research Institute Funded Ministry of Korea Small and Medium Venture Business in 2017.

\section{References}

[1] J. S. Kim, J. Jeong, and T. S. Chung, "Development of the IoT-based remote monitoring system of sleeping hours," in Proceedings of the 2019 4th International Conference on Intelligent Information Technology, Da Nang, Vietnam, 2019, pp. 42-45. http://10.1145/3321454.3321463

[2] K. S. Min, "Internet of Things," 2013, Available http://www.kisa.or.kr/uploadfile/201306/20130610174053 1675.pdf

[3] N. McCarthy, "How prevalent is smart technology in U.S. homes?," 2015, Available https://www.forbes.com/ sites/niallmccarthy/2015/10/06/how-prevalent-is-smarttechnology-in-u-s-homes-infographic/\#325e3d22eef6

[4] IoT Agenda, "Smart home or building (home automation or domotics)," 2018; https://internetofthingsagenda. techtarget.com/definition/smart-home-or-building
[5] J. H. Jeong, et al., "Implementation and design of the REST API off smart mat system for remote monitoring," in Proceedings of the Korea Multimedia Society Fall Conference, Seoul, Korea, 2018.

[6] S. Bhardwaj, T. Ozcelebi, J. J. Lukkien, and K. M. Lee, "Semantic interoperability architecture for smart spaces," International Journal of Fuzzy Logic and Intelligent Systems, vol. 18, no. 1, pp. 50-57, 2018. https: //doi.org/10.5391/IJFIS.2018.18.1.50

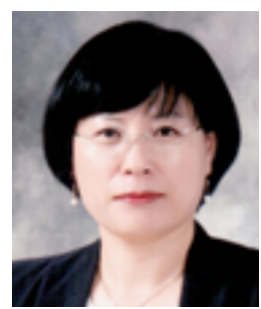

Jung-Sook Kim is a professor at the School of CIT Convergence, Kimpo University, Korea. She received her B.S., M.S., and Ph.D. degrees in computer engineering from Dongguk University, Seoul, Korea in 1993, 1995, and 1999, respectively. Her research interests include the fields of intelligent systems, IT convergence, and distributed and parallel systems. E-mail: kimjs@kimpo.ac.kr

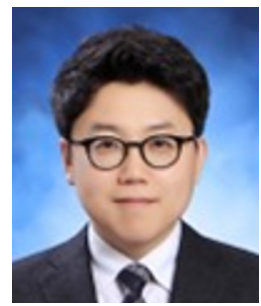

Junho Jeong received the B.S. degree from the Dept. of Computer Science and Engineering, Dongguk University, Seoul, Korea, in 2007, and M.S. and Ph.D. degrees from the Dept. of Computer Science and Engineering, Dongguk University, Seoul, Korea in 2009 and 2015, respectively. He was a research professor of Electronic Commerce Institute, Dongguk University, Gyeongju, Korea from 2015-2019. And he was a research professor of Dept. of Computer Science and Engineering, Dongguk University, Seoul, Korea until 2019 Aug. Currently, he is an assistant professor of the Dept. of Computer Science and Engineering, Kongju National University, Cheonan, Korea. His research areas include Computer Security, Privacy Preserving, Distributed System, Network Security and Secure Software.

E-mail: yanyenli@kongju.ac.kr 